\title{
Aspectos biológicos de Coccidophilus citricola (Coleoptera, Coccinellidae) sobre Aspidiotus nerii e Chrysomphalus aonidum (Homoptera, Diaspididae) ${ }^{1}$
}

\author{
Biological studies of Coccidophilus citricola (Coleoptera, Coccinellidae) on Aspidiotus nerii and \\ Chrysomphalus aonidum (Homoptera, Diaspididae)
}

\author{
Antonio Cesar dos Santos ${ }^{2}$ Santin Gravena ${ }^{3}$
}

\section{RESUMO}

Aspectos biológicos de Coccidophilus citricola Brèthes (Coleoptera, Coccinellidae) foram estudados sobre Chrysomphalus aonidum Linnaeus e Aspidiotus nerii Bouchè (Homoptera, Diaspididae) criadas sobre abóbora. Observouse a duração (dias) do período de ovo, dos quatro ínstares, pré-pupa, pupa, longevidade de adultos e a proporção machos: fêmeas de C. citricola. Determinou-se também verificar a eficiência de predação de adultos de C. citricola sobre A. nerii. C. aonidum e A. nerii são presas adequadas para C. citricola, com desenvolvimento semelhante do coccinelídeo sobre essas cochonilhas; o período de incubação do ovo foi de 7,9 e 8,1 dias com A. nerii e C. aonidum; a duração (dias) de cada ínstar larval de $\boldsymbol{C}$. citricola sobre $\boldsymbol{A}$. nerii e C. aonidum foram respectivamente de: (I) 2,7 e 2,9; (II) 3,1 e 3,3; (III) 3,0 e 3,3 e (IV) 3,2 e 3,1 dias. A duração das fases de pré-pupa e pupa foi de 2,5 e 4,5 dias sobre A. nerii e 2,8 e 4,8 dias sobre C. aonidum e a viabilidade de ovo a adulto foi de $63,2 \%$ e $62,7 \%$ respectivamente para indivíduos criados sobre essas presas. A longevidade de adultos de C. citricola foi de 57,0 dias com A. nerii e de 62,0 dias com C. aonidum. A proporção macho: fêmea foi de 0,82:1,00. O consumo diário de C. citricola foi de 1,15 adultos ou 2,35 ninfas de 2o ínstar ou 3,16 ninfas de 1ํ́instar de A. nerii por dia.

Palavras-chave: Insecta, cochonilhas, coccinelídeos, inimigo natural, insetos predadores.

\section{ABSTRACT}

Laboratory trials were carried out to study biological aspects of Coccidophilus citricola Brèthes
(Coleoptera: Coccinellidae) on Chrysomphalus aonidum Linnaeus and Aspidiotus nerii Bouché (Homoptera: Diaspididae). The scales were reared on squashes and then served as food source to the predator. The period of egg, larvae (four ínstars), pre-pupae, pupae, adults' longevity and male: female ratio was observed. Predatory efficacy of C. citricola on A. nerii was also determined. The period of egg was 7.9 and 8.1 days on $A$. nerii and $C$. aonidum. The development (days) of different stages of $\boldsymbol{C}$. citricola on $\boldsymbol{A}$. nerii or C. aonidum were similar. The period of each larval ínstar on A. nerii and C. aonidum, respectively was (I) 2.7 and 2.9; (II) 3.1 and 3.3; (III) 3.0 and 3.3; (IV) 3.2 and 3.1 days. Duration of pre-pupae stage was 2.5 and 2.8 days and pupae stage was 4.5 and 4.8 days on $A$. nerii and $C$. aonidum. The viability from egg to adult was $63.2 \%$ and $62.7 \%$ respectively on $A$. nerii and C. aonidum. Adults' longevity was 57 and 62 days on A. nerii and C. aonidum. Male: female ratio was 0.82: 1.00. Consumption of adults of C. citricola on A. nerii was 1.15 adults or 2.35 nymphs of $2^{\text {nd }}$ ínstar or 3.16 nymphs of $1^{\text {st }}$ ínstar / day.

Key words: Insecta, scales, coccinellids, natural enemies, predatory insects.

\section{INTRODUÇÃO}

O setor citrícola ocupa lugar de destaque na economia agrícola brasileira como fonte de geração de divisas, renda interna, emprego e contribuição em termos nutricionais (NEVES, 1992). Em 2001/02, a

\footnotetext{
${ }^{1}$ Parte da dissertação apresentada pelo primeiro autor ao Programa de Pós-graduação em Entomologia, Departamento de Fitossanidade, Faculdade de Ciências Agrárias e Veterinárias (FCAV), Universidade Estadual Paulista (UNESP), Jaboticabal, SP, para obtenção do título de Mestre. Projeto financiado pela Fundação de Amparo a Pesquisa do Estado de São Paulo (FAPESP).

${ }^{2}$ Engenheiro Agrônomo, MSc, Doutorando da Faculdade de Filosofia Ciências e Letras (FFCL), Universidade de São Paulo (USP), Ribeirão Preto, SP. Rua Dr. Raul R. Medeiros, 257, 15910-000, Monte Alto, SP. E-mail: acsantos1@dow.com. Autor para correspondência.

${ }^{3}$ Engenheiro Agrônomo, PhD, Gravena Manejo Ecológico de Pragas, Rod. SP 253, Km 221,5, CP 546, 14870-990, Jaboticabal, SP. E-mail: gravena@gravena.com.br.
} 
produção atingiu 361,7 milhões de caixas em 187,8 milhões de plantas (AMARO, 2003). No entanto, alguns fatores como as cochonilhas Selenaspidus articulatus Morgan, Parlatoria ziziphus Lucas, Unaspis citri Comstock, Parlatoria cinerea Hadden e Chrysomphalus aonidum Linnaeus, podem limitar a produção citrícola (GRAVENA, 1990).

As joaninhas são predadores de insetos, porém métodos para aumentar e conservar suas populações em campo são ainda pouco desenvolvidos (OLKOWSKI et al., 1990). A eficiência desses inimigos naturais mostra que devem ser incorporados à programas de Manejo Integrado de Pragas (HODEK, 1973). Coccidophilus citricola Bréthes (Coleoptera: Coccinellidae) é um importante predador de várias cochonilhas na Argentina, Chile, Uruguai, Paraguai e Brasil atacando principalmente Cornuaspis beckii Newmann, C. aonidum, Chrysomphalus dictyospermi Morgan, Aonidiella aurantii Maskell, entre outras (BOSQ, 1943). C. citricola foi uma das espécies mais abundantes em citros na Argentina, o que mostra sua importância para programas de introdução e incremento de inimigos naturais CROUZEL (1973).

Este trabalho teve por objetivo estudar aspectos biológicos sobre as cochonilhas da família Diaspididade, A. nerii e $\boldsymbol{C}$. aonidum e a eficiência de predação da joaninha sobre $\boldsymbol{A}$. nerii.

\section{MATERIAL E MÉTODOS}

Abóboras infestadas com $\boldsymbol{A}$. nerii ou $\boldsymbol{C}$. aonidum foram cortadas em pedaços de aproximadamente $4 \mathrm{x} 4 \mathrm{~cm}$, parafinados na sua parte inferior para evitar o rápido apodrecimento e colocados, logo após o resfriamento, em recipiente plástico (15 x $20 \mathrm{~cm}$ ) com 100 adultos de $\boldsymbol{C}$. citricola. A porção superior desse recipiente foi fechada com tecido tipo voil, permitindo a passagem de luz e ar. Um dia após, os pedaços de abóbora foram retirados do recipiente e observados sob microscópio estereoscópico. As carapaças das cochonilhas foram levantadas com um estilete para se observar a presença de ovos de $\boldsymbol{C}$. citricola, pois o predador oviposita sob as mesmas. Os ovos encontrados foram transferidos, com um pincel fino e umedecido, para pequenas arenas constituídas de seções de $1,5 \mathrm{~cm}$ de mangueira plástica transparente com $1,5 \mathrm{~cm}$ de diâmetro com o fundo constituído de papel filtro colado na mesma. Colocados os ovos nas arenas, estas foram fechadas com plástico tipo “magipack”. Diariamente os ovos foram observados sob microscópio estereoscópico até a eclosão, obtendo-se o período embrionário.
Estudou-se a biologia de $\boldsymbol{C}$. citricola com A. nerii e posteriormente com C. aonidum. Obteve-se o período larval total e a duração de cada ínstar, além da duração e viabilidade das fases de pré-pupa e pupa, do ciclo de vida e a proporção machos: fêmeas. Adultos de $\boldsymbol{C}$. citricola foram alimentados com cochonilhas até a morte dos mesmos. Pela dificuldade de determinação dos sexos visualmente (FLANDERS, 1936 e GORDON, 1978), utilizou-se um método de dissecação de espécimes. Cinquenta indivíduos foram colocados em tubos de ensaio com hidróxido de potássio a $10 \%$ e após cinco minutos colocados em água fervente por 15 minutos. Esse indivíduos foram dissecados para retirada da genitália para diferenciarse machos e fêmeas. Foram utilizados de 30 a 100 indivíduos para obtenção dos dados em cada fase (incubação dos ovos, larva I, II, III e IV, pré-pupa, pupa e adulto) sendo os mesmos submetidos à análise de variância e ao teste $\mathrm{F}$.

A predação de adultos de $\boldsymbol{C}$. citricola foi obtida sobre ninfas e adultos de $\boldsymbol{A}$. nerii. Cinco pedaços de abóbora, cada um considerado como uma repetição, infestados com ninfas de primeiro ínstar, outros cinco com ninfas de segundo ínstar e mais cinco com adultos da cochonilha foram colocados separadamente em placas de Petri. Em cada uma das placas, foram colocadas 10 joaninhas. O número de diaspidídeos predados foi obtido após 24 horas. Em outro teste, adultos de $\boldsymbol{C}$. citricola foram colocados sobre pedaços de abóboras infestados com todos os ínstares de $\boldsymbol{A}$. nerii, para se observar a preferência de C. citricola por determinado ínstar da cochonilha.

As condições do estudo em laboratório foram $25^{\circ} \pm 2^{\circ} \mathrm{C}, 70 \pm 10 \%$ de UR e fotofase de 12 horas.

\section{RESULTADOS E DISCUSSÃO}

O período embrionário de $\boldsymbol{C}$. citricola foi de 7,9 e 8,1 dias com $\boldsymbol{A}$. nerii e $\boldsymbol{C}$. aonidum, com diferença significativa entre esses valores (Tabela 1), enquanto a viabilidade desta fase foi de $77,6 \%$ e $79,3 \%$ respectivamente com $\boldsymbol{A}$. nerii e $\boldsymbol{C}$. aonidum. Fêmeas de $\boldsymbol{C}$. citricola ovipositam geralmente de 1 a 3 (1,4 \pm $0,67)$ ovos. Preferencialmente os ovos são colocados sob carapaças vazias, sem a presença de cochonilhas (68,3\%), mas podem também ser colocados em carapaças com cochonilhas ainda vivas $(31,7 \%)$. A oviposição de $\boldsymbol{C}$. citricola foi semelhante sob carapaças de cochonilhas adultas $(48,4 \%)$ ou ninfas de segundo ínstar (51,6\%), em sua maioria em carapaças com sinal de predação (76,9\%). Fêmeas desse predador procuram ovipositar em locais com grande 
Tabela 1 - Duração (dias) dos diferentes estádios de Coccidophilus citricola Brèthes (Coleoptera: Coccinellidae) sobre Aspidiotus nerii Bouchè e Chrysomphalus aonidum Linnaeus (Homoptera: Diaspididae) em condições de laboratório $\left(\mathrm{T}=25 \pm 2^{\circ} \mathrm{C}, \mathrm{UR}=70 \pm\right.$ $10 \%$ e Fotofase: $12 \mathrm{~h})$.

\begin{tabular}{|c|c|c|c|c|c|c|}
\hline \multirow[b]{2}{*}{ Estádio } & \multirow[b]{2}{*}{ (n) } & \multicolumn{2}{|c|}{ A. nerii } & \multicolumn{2}{|c|}{ C. aonidum } & \multirow[b]{2}{*}{$\mathrm{CV}$} \\
\hline & & Duração & $\mathrm{IV}^{2}$ & Duração & $\mathrm{IV}^{2}$ & \\
\hline Período embrionário ${ }^{1}$ & 100 & $7,9 \pm 0,82$ & $7-9$ & $8,1 \pm 0,61$ & $7-9$ & 9,03 \\
\hline Larva I & 50 & $2,7 \pm 0,95$ & $1-4$ & $2,9 \pm 0,82$ & $1-4$ & 3,50 \\
\hline Larva II & 50 & $3,1 \pm 0,55$ & $2-4$ & $3,3 \pm 0,73$ & $2-4$ & 20,80 \\
\hline Larva III & 50 & $3,0+0,64$ & $2-4$ & $3,3 \pm 0,84$ & $2-4$ & 24,85 \\
\hline Larva IV & 50 & $3,2 \pm 0,50$ & $2-4$ & $3,1 \pm 0,83$ & $2-4$ & 19,75 \\
\hline Pré-Pupa & 50 & $2,5 \pm 0,45$ & $1-4$ & $2,8 \pm 0,72$ & $2-4$ & 18,25 \\
\hline Pupa & 50 & $4,5 \pm 0,57$ & $3-6$ & $4,8 \pm 0,70$ & $4-6$ & 17,50 \\
\hline Longevidade $^{1}$ & 30 & $57,0 \pm 7,31$ & $47-67$ & $62,0 \pm 6,70$ & $50-70$ & 12,30 \\
\hline
\end{tabular}

$\mathbf{1}=$ fases onde houve diferença estatística pelo Teste F.

${ }^{2}=$ Intervalo de variação.

concentração da presa, ou seja, em colônias (83,8\%) em detrimento de carapaças isoladas (16,2\%), pois suas larvas permanecem debaixo das carapaças se alimentando durante os primeiros e segundo ínstares.

C. citricola apresenta quatro ínstares com a seguinte duração sobre $\boldsymbol{A}$. nerii e $\boldsymbol{C}$. aonidum, respectivamente: (I) 2,7 e 2,9; (II) 3,1 e 3,3; (III) 3,0 e 3,3 e (IV) 3,2 e 3,1 (Tabela 1). ALMEIDA (1990), estudando a biologia de $\boldsymbol{C}$. citricola à temperatura de $30,44^{\circ} \mathrm{C}$ e UR de $78 \%$, sobre Diaspis echinocacti Bouché, apontou os seguintes resultados: período embrionário $(8,14)$; larva I $(2,66)$; larva II $(1,74)$; larva III $(2,22)$ e larva IV (2,52). Isto mostra o efeito da temperatura pois o desenvolvimento de formas imaturas em Coccinellidae pode variar com as mesmas HAGEN (1962). A viabilidade de cada ínstar foi de $89,0,94,7,100,0$ e de $100,0 \%$ e 86,2, 95,0, 100,0 e 100,0\% no primeiro, segundo, terceiro e quarto ínstares com A. nerii e $\boldsymbol{C}$. aonidum respectivamente.

A fase de pré-pupa de $\boldsymbol{C}$. citricola durou 2,5 e 2,8 dias com $\boldsymbol{A}$. nerii e $\boldsymbol{C}$. aonidum, sem diferença significativa (Tabela 1), com viabilidade de 98,3 e $98,0 \%$, respectivamente. A fase pupal durou 4,5 e 4,8 dias com viabilidade de $98,3 \%$ e $98,5 \% \operatorname{com} A$. nerii e C. aonidum, também sem diferença significativa. A viabilidade de ovo a adulto foi de $63,2 \%$ e $62,7 \%$ para indivíduos criados sobre $\boldsymbol{A}$. nerii e $\boldsymbol{C}$. aonidum, com maior valor que os resultados para $D$. enchinocacti (ALMEIDA, 1990),

Adultos de $\boldsymbol{C}$. citricola apresentam, logo após a emergência, coloração amarelo-amarronzada, passando a preto após alguns minutos, de forma semelhante ao descrito por BRÈTHES (1905). A longevidade de seus adultos foi de 57,0 e 62,0 dias com A. nerii e $\boldsymbol{C}$. aonidum. A longevidade de machos
(65,4 dias) e fêmeas (65,6 dias) sobre $\boldsymbol{C}$. aonidum foi semelhante. Obteve-se a proporção macho: fêmea que foi de 0,82:1,00, que diferiu de ALMEIDA (1990), 0,92:1,00, para indivíduos do campo.

C. citricola teve predação diária de $1,15 \pm 0,42$ adultos ou 2,35 $\pm 0,96$ ninfas de segundo ínstar ou 3,16 $\pm 1,84$ ninfas de primeiro ínstar de $\boldsymbol{A}$. nerii/dia com valores maiores que os de $\boldsymbol{C}$. citricola sobre $\boldsymbol{D}$. echinocacti de 0,45 cochonilha/dia (ALMEIDA 1990), com fornecimento de pedaços de palma forrageira com cochonilhas de todos os ínstares. Um total de 22,5\% das carapaças com sinal de predação apresentaram cochonilhas adultas intactas mas, geralmente, existiam ovos e ninfas de primeiro ínstar, ou restos desses sob as carapaças. Isto pode indicar que o predador ao perfurar a carapaça, pode se alimentar de ovos e ninfas, ao invés da cochonilha adulta. A baixa predação de C. citricola em adultos de $\boldsymbol{D}$. echinocacti se dá, principalmente, pela preferência por ovos (ALMEIDA 1990). Além disso, 45,5\% dos diaspidídeos predados estavam apenas danificados, mostrando que o coccinelídeo alimenta-se apenas de parte da presa. Em $32 \%$ das carapaças com sinal de predação, não havia cochonilhas. Em relação à preferência na predação, 78,1\% foram ninfas e 21,9\% adultos. A baixa eficiência de $\boldsymbol{C}$. citricola sobre cochonilhas adultas (COMPERE, 1961; ALMEIDA, 1990), pode ser compensada pelo fato desta joaninha se alimentar em grande parte de formas jovens (ovos e ninfas de primeiro e segundo ínstares).

\section{CONCLUSÃO}

C. citricola apresenta duração (dias) e viabilidade das diferentes fases semelhantes sobre $\boldsymbol{A}$. 
nerii ou $C$. aonidum, consumindo em maior número , estágios imaturos de $\boldsymbol{A}$. nerii.

\section{REFERÊNCIAS BIBLIOGRÁFICAS}

ALMEIDA, R.P. Aspectos bioecológicos de predadores (Coleoptera: Coccinellidae) sobre a cochonilha da palma forrageira Diaspis echinocacti Bouché, 1833 (Homoptera: Diaspididae) em condições de laboratório. 1990. $138 f$. Dissertação (Mestrado em Entomologia) - Universidade Federal Rural de Pernambuco.

AMARO, A.A. Projeções de produção de laranja em São Paulo até 2009. Capturado em 10 dez. 2003. Online. Disponível na Intenernet: http// www.iea.sp.gov.br

BOSQ, J.M. Coccinelídeos utiles para la fruticultura tucumana. Rev Soc Entomol Arg, v.11, p.461-470, 1943.

BRÈTHES, J. Descripcion de un genero y de una nueva especie de clavicornio de Buenos Aires (Coleoptero). Anal Soc Cient Arg, v.59, p.76-79, 1905

COMPERE, H. The red scale and its insects enemies. Hilgardia, v.31, p.173-278, 1961.
CROUZEL, I.S. Estudo sobre control biologico de cochonilhas Diaspididae que atacam citricos en la Republica Argentina. IDIA, v.304, p.15-39, 1973

FLANDERS, S.E. Coccidophilus citricola Brèthes, a predator enemy of red and purple scales. J Econ Entomol, v.29, p.10231024, 1936.

GORDON, R.D. West Indian Coccinellidae II (Coleoptera): Some scale predators with Key to genera and species. Colept Bull, v.32, p.205-218, 1978

GRAVENA, S. Manejo integrado de pragas de citros na atualidade. In: SIMPÓSIO DE MANEJO INTEGRADO DE PRAGAS, 1., 1990, Jaboticabal, SP. Anais... Jaboticabal : FUNEP, 1990. V.1, 253p, p.107-126.

HAGEN, K.S. Biology and ecology of predaceous Coccinellidae. Ann Rev Entomol, v.7, p.289-326, 1962.

HODEK, I. Biology of Coccinellidae. Prague: Czechoslovak Academy of Sciences, 1973. 260p.

NEVES, E.M. Citricultura brasileira: importância econômica e perspectivas. Laranja, v.13, p.55-62, 1992.

OLKOWSKI, W. et al. Improved biocontrol techniques with lady beetles. IPM-Practioner, v.12, p.1-12., 1990. 